\title{
Molecular dynamics simulations of valinomycin interactions with potassium and sodium ions in water solvent
}

\author{
Kholmirzo Kholmurodov ${ }^{1,2}$, Maria Abasheva ${ }^{2}$, Kenji Yasuoka ${ }^{3}$ \\ ${ }^{1}$ Laboratory of Radiation Biology, Joint Institute for Nuclear Research, Dubna, Russia; \\ ${ }^{2}$ Dubna International University, Dubna, Russia; \\ ${ }^{3}$ Department of Mechanical Engineering, Keio University, Yokohama, Japan. \\ Email: mirzo@jinr.ru
}

Received 17 June 2010; revised 25 June 2010; accepted 26 June 2010.

\begin{abstract}
The aim of this work is to estimate the value of the electric field (potentials) for the system of valinomycin $+\mathrm{K}^{+}$and $\mathrm{Na}^{+}$ions based on a molecular dynamics (MD) study. An analysis has been performed of the interaction processes for the system of valinomycin + $\mathrm{K}^{+}\left(\mathrm{Na}^{+}\right)$ion in water solvent. It is obtained that capturing a $\mathrm{K}^{+}\left(\mathrm{Na}^{+}\right)$ion in the valinomycin cavity is not possible for all values of the electric field strength. Each of the two kinds of ions $\left(\mathrm{K}^{+}\right.$or $\left.\mathrm{Na}^{+}\right)$has its own critical electric field associated with ion binding to valinomycin, for which to exist, the ion has to remain localized inside the valinomycin cavity. The results obtained for the electrical potential reveal a stronger valinomycin binding-especially with the potassium ion. Valinomycin's molecular structure efficiently surrounds the $\mathrm{K}^{+}$ion, as this structure has to exactly correspond to the $\mathrm{K}^{+}$ion in size. MD simulation results could be a prerequisite for studying a more complex scenario-for estimating ion transport in the cell membrane or physiological electric potential which is formed in the membrane or inside the cell relative to its surrounding medium.
\end{abstract}

Keywords: Molecular Dynamics Simulations; Valinomycin; Potassium and Sodium Ions

\section{INTRODUCTION}

Valinomycin was extracted for the first time from Streptomyces fulvissimus bacteria in 1955; in 1967, it was established that as a transporter, valinomycin catalyzes the exchange of $\mathrm{K}^{+}$and $\mathrm{H}^{+}$through a mitochondrial cell membrane, thereby causing no changes in the $\mathrm{Na}^{+}$concentration [1-3]. In biological membranes, there are several kinds of ionic pumps, which work at the expense of the free energy of ATP hydrolysis - a special $\mathrm{Na}^{+} / \mathrm{K}^{+}$-ATPase system of integrated proteins known as the sodium-potassium pump. The ATPase mechanism of ion transport is realized as a transfer process conjugated with chemical reactions, which goes at the expense of the cell metabolism energies. During the functioning of the $\mathrm{Na}^{+} / \mathrm{K}^{+}$-ATPase at the expense of the chemical binding energeis released in the hydrolysis of each ATP molecule, two sodium ions transfer into the cell with the simultaneous extraction of three potassium ions. Thus, an electric potential gradient is formed due to an increase in the concentration of potassium ions in comparison to that of the interstitial media and a decrease in the sodium concentration, which is physiologically important.

Specifically in neurons, the combination of the two mechanisms mentioned above corresponding to their state of rest is responsible for dynamical equilibrium stability. The internal sodium concentration in cells is ten times lower than in surrounding media; at the same time, the potassium concentration is ten times higher. Such a disbalance tends to equilibrate the streams going through narrow pores of the cell membrane. To control the necessary ion concentration in the cell, the membrane protein molecules (called "sodium pumps") continuiously pump potassium from, and sodium into, the cell. Each pump is able to transfer about 200 sodium ions and 130 potassium ions per second. A neuron, for example, does about a million of such pumps that transfer hundreds of millions of potassium and sodium ions through a cell membrane per second [2-4].

The potassium concentration in the cell is influenced by a large number of open potassium chanells (i.e. protein molecules), which allow potassium ions to pass easily into the cell but suppress sodium ions passing through. For the transfer of potassium ions and other particles into the cell, special membrane transport proteins must be responsible. Valinomycin is an example of a transporter protein for potassium ions. Valinomycin has a macrocyclic (ring) structure as shown in Figures 1(a) and (b). The valinomycin molecule is highly selective to potassium ions as compared to sodium ions 


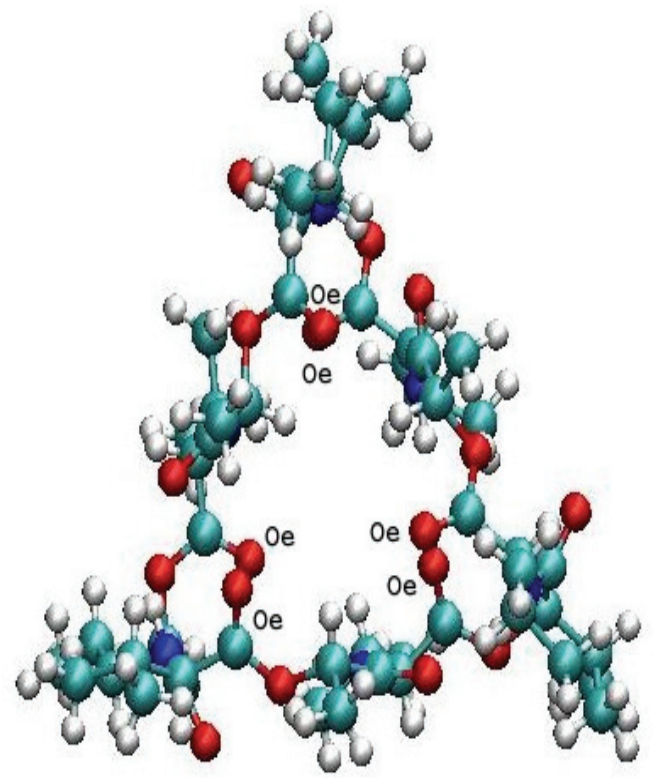

(a)

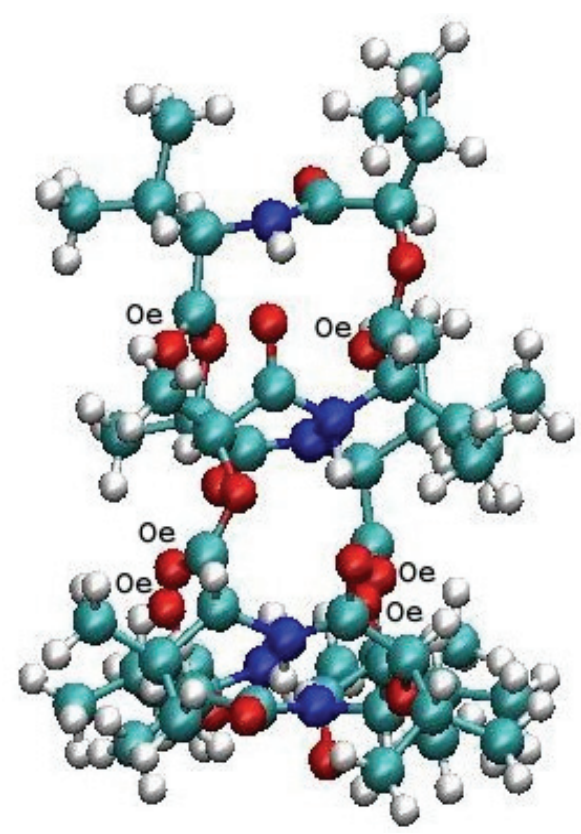

(b)

Figure 1. The valinomycin configuration. (a) a view from the molecule plane; (b) a side view. The color spheres represent nitrogen (blue), carbon (blue), hydrogen (white), and oxygen (red) atoms. The six oxygen atoms which are able to capture external solvent ions are denoted as Oe.

within the cell membrane; it has 12 carbonyl groups for the binding of metal ions, and also for solvation in a polar solvent. The isopropyl groups and methyl groups are responsible for solvation in nonpolar solvents. Along with the molecule's shape and size, this molecular duality is the main reason for its binding properties. In polar solvents, valinomycin will mainly expose the carbonyls to the solvent; in nonpolar solvents, the isopropyl groups are located predominantly on the exterior of the molecule. This conformation will change as valinomycin binds to a potassium ion. The molecule is "locked" into a conformation with the isopropyl groups on the exterior. Due to its chemical structure, valinomycin is able to form a complex with a potassium ion captured by the molecule - inside its ring; on the other hand, valinomycin is easily solvable in the membrane lipid phase - it is non-polar on the exterior part. Thus, the valinomycin molecules that are positioned on a membrane surface capture potassium ions from the surrounding solvent; then potassium ions are transferred by valinomycin by means of diffusion in the membrane, and, finally, ions are released in the solvent on the other side of the cell membrane. A gradient of the ion concentration in the cell membrane is thereby created; an electric potential relative to the cell surrounding varies from $-70 \mathrm{mV}$ to $+50 \mathrm{mV}$. The potential stimulates a synaptic signal transmission necessary for biological functions [3-6].

In this work, based on the molecular dynamics (MD) simulation, we aimed to measure the electric field strength (potential gradients) for the model systems describing valinomycin with potassium $\left(\mathrm{K}^{+}\right)$and sodium $\left(\mathrm{Na}^{+}\right)$ions. To calculate electrostatics interactions, we used a reaction field algorithm [7]. We performed an $\mathrm{MD}$ analysis in comparison with the physiological data on the cell electric potential outlined above. It should be noted that MD simulation is one of the most applicable techniques used to study valinomycin's dynamical and equilibrium properties in biological systems. For the valinomycin interaction with different cations and solutions, see, for example, references [8-10]. In [8], valinomycin selectivity for the transport of potassium ions (and not sodium ions) is studied by MD simulation. The process of potassium ion capture by a valinomycin molecule is illustrated in [9]. An estimation of the energy of the cation binding to valinomycin is performed in [10].

\section{MATERIALS AND METHODS}

The starting configuration of the molecular system consisting of valinomycin with potassium ions is shown in Figure 1. We have used periodic boundary conditions for all spatial axes; the geometry of the system configuration was a truncated octahedron with the side length of $42,86 \AA$. Molecular dynamics (MD) simulations have been performed using the DL_POLY code, which was developed by the molecular simulation group at the Daresbury Laboratory (England) with the support of the 


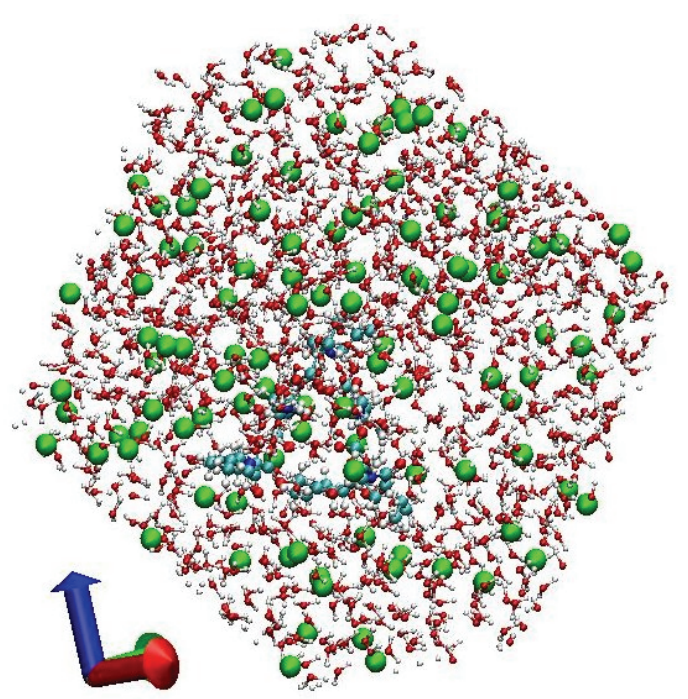

Figure 2. A valinomycin molecule (a triangular shape chain is in the center) surrounded by potassium ions (green spheres) and water molecules (red and white are oxygens and hydrogens, respectively).

Research Council for Engineering and Physical Sciences (project CCP5 for simulating condensed phases). DL_POLY is a general-purpose MD simulation package developed by W. Smith, T.P. Forester, and I.T. Todorov [11]. We have employed version 2.19 of the DL_POLY; the initial geometry of the biphenyl molecule was chosen from the database of the program package at:

http://www.cse.scitech.ac.uk/ccg/software/DL POLY/

The configurational energy of the molecular model is represented as a sum of the energies of binding $\left(\mathrm{E}_{\mathrm{val}}\right)$ and non-binding $\left(\mathrm{E}_{\mathrm{nb}}\right)$ interactions:

$$
\mathrm{E}=\mathrm{E}_{\mathrm{val}}+\mathrm{E}_{\mathrm{nb}} \text {. }
$$

The energy of valence (binding) interactions $\mathrm{E}_{\mathrm{val}}$ is given by the following formula:

$$
E_{\mathrm{val}}=\mathrm{E}_{\mathrm{imb}}+\mathrm{E}_{\mathrm{ang}}+\mathrm{E}_{\mathrm{dih}}+\mathrm{E}_{\mathrm{inv}},
$$

where $E_{i m b}$ is the energy of intermolecular bonds, $E_{\text {ang }}$ is the energy of angular bonds, $\mathrm{E}_{\mathrm{dih}}$ is the energy of dihedral bonds, and $\mathrm{E}_{\text {inv }}$ is inversion energy.

The energy of the non-valence (non-bound) interactions is a sum of the energies of the van der Waals (vdW), electrostatics (Coulomb), and hydrogen bonds:

$$
\mathrm{E}_{\mathrm{nb}}=\mathrm{E}_{\mathrm{vdW}}+\mathrm{E}_{\text {coul }}+\mathrm{E}_{\mathrm{hb}} \text {. }
$$

The valinomycin molecule consists of 168 atoms; the number of $\mathrm{K}^{+}\left(\mathrm{Na}^{+}\right)$ions was 109 . The water molecules were simulated as 3-site rigid bodies; the total number of water atoms was $3339(1113 \times 3)$. Computer simulations were performed for a constant temperature of $300 \mathrm{~K}$ using the Nose - Hoover algorithm with the thermostat relaxation constant of 2 ps. For the van der Waals interactions, we have used the Lennard - Jones (LJ) potential. The interaction potential parameters and atomic masses and charges are shown in Tables 1 and 2. The integration of the equations of motion was performed using the Verle integration scheme in quaternion. The integration step was 2 fs (femtoseconds). The intermolecular chemical bonds were estimated on the basis of the Shake algorithm to an accuracy of $10^{-8}$.

The electrostatics forces were calculated using the socalled "reaction field" algorithm [7,11]. In this method, the molecule is surrounded by a spherical cavern of a limited radius where the electrostatics forces are calculated directly. Outside of the cavern, the system is represented as a dielectric continuum. In the reaction field algorithm, the Coulomb potential has the following form:

Table 1. The Lennard - Jones (LJ) potential parameters for different atomic pairs.

\begin{tabular}{|c|c|c|c|c|c|}
\hline Atomic pair & Potential & Functional form & Parameters & $\boldsymbol{\varepsilon}, \mathbf{k c a l} / \mathbf{m o l}$ & $\mathbf{\sigma}, \boldsymbol{\AA}$ \\
\hline C-C & $L J$ & $4 \varepsilon\left[\left(\frac{\sigma}{r}\right)^{12}-\left(\frac{\sigma}{r}\right)^{6}\right]$ & $\boldsymbol{\varepsilon}, \boldsymbol{\sigma}$ & 0.12 & 3.30 \\
& & $\ldots$ & & & \\
H-H & $\ldots$ & $\ldots$ & $\ldots$ & 0.02 & 1.78 \\
N-N & $\ldots$ & $\ldots$ & $\ldots$ & 0.16 & 3.12 \\
O-O & $\ldots$ & $\ldots$ & $\ldots$ & 0.20 & 2.85 \\
OS-OS & $\ldots$ & $\ldots$ & $\ldots$ & 0.15 & 2.94 \\
Oe-Oe & $\ldots$ & $\ldots$ & $\ldots$ & 0.20 & 2.85 \\
OW-OW & $\ldots$ & $\ldots$ & $\ldots$ & 0.02 & 3.17 \\
HW-HW & $\ldots$ & $\ldots$ & $\ldots$ & 0.32 & 3.78 \\
K-K & $\ldots$ & $\ldots$ & $\ldots$ & 0.08 & 2.73 \\
Na-Na & $\ldots$ & $\ldots$ &
\end{tabular}


Table 2. The mass and charge values in the system of valinomycin $+\mathrm{K}^{+}\left(\mathrm{Na}^{+}\right)$ion + water.

\begin{tabular}{ccc}
\hline $\begin{array}{c}\text { Atom } \\
(\mathrm{md} \\
\text { notation })\end{array}$ & $\begin{array}{c}\text { Mass } \mathrm{m} \\
\left(\mathrm{m}_{\mathrm{e}}, \text { a.m.u. }\right)\end{array}$ & $\begin{array}{c}\text { Charge } \mathrm{q} \\
\text { (e, proton charge })\end{array}$ \\
\hline $\mathrm{C}$ & 12.01 & +0.47 \\
$\mathrm{H}$ & 1.00 & +0.21 \\
$\mathrm{~N}$ & 14.01 & -0.40 \\
$\mathrm{O}$ & 16.00 & -0.41 \\
$\mathrm{OS}$ & 16.00 & -0.46 \\
$\mathrm{Oe}$ & 16.00 & -0.41 \\
$\mathrm{OW}$ & 15.99 & -0.82 \\
$\mathrm{HW}$ & 1.00 & +0.41 \\
$\mathrm{~K}$ & 39.10 & +1.00 \\
$\mathrm{Na}$ & 23.00 & +1.00 \\
\hline
\end{tabular}

$$
U\left(r_{n j}\right)=\frac{1}{4 \pi \varepsilon_{0}} q_{j} q_{n}\left[\frac{1}{r_{n j}}+\frac{B_{0} r_{n j}{ }^{2}}{2 R_{c}^{3}}\right],
$$

where $R_{c}$ is a cavern radius, the constant $B_{0}$ is the dielectric constant of the continuum media, and

$$
B_{0}=\frac{2\left(\varepsilon_{1}-1\right)}{\left(2 \varepsilon_{1}+1\right)} .
$$

The non-bound vdW forces are calculated using the LJ potential of the standard form:

$$
U(r)=4 \varepsilon\left[\left(\frac{\sigma}{r}\right)^{12}-\left(\frac{\sigma}{r}\right)^{6}\right]
$$

For different atoms, we applied the following averaged relations (the Lorentz-Berthelot combining rules):

$$
\varepsilon_{i j}=\left(\varepsilon_{i i} \varepsilon_{j j}\right)^{\frac{1}{2}} \text { and } \sigma_{i j}=\frac{1}{2}\left(\sigma_{i i}+\sigma_{i j}\right) .
$$

In Table 2, the mass and charge values are presented for the system of valinomycin $+\mathrm{K}^{+}\left(\mathrm{Na}^{+}\right)$ion + water used in molecular dynamics simulations.

\section{RESULTS AND DISCUSSIONS}

We have fulfilled a series of MD calculations for the systems of valinomycin $+\mathrm{K}^{+}$ions + water and valinomycin $+\mathrm{Na}^{+}+$water with the same simulation parameters and temperature-pressure conditions as described above. In order to control the motion of $\mathrm{K}^{+}\left(\mathrm{Na}^{+}\right)$ ions directed exactly to the valinomycin cavity (ring), an external electric field of different fixed strength values was applied. Without an external field, valinomycin's interaction with $\mathrm{K}^{+}\left(\mathrm{Na}^{+}\right)$ions takes place only in the vicinity of the molecule, but the ions do not enter the cavity itself. In Figures 3(a) and (b), we present the initial configuration of the valinomycin $+\mathrm{K}^{+}$ions, where the electric field is directed normally to the molecule plane (water molecules are not shown). The orientation of valinomycin during the whole time of dynamical

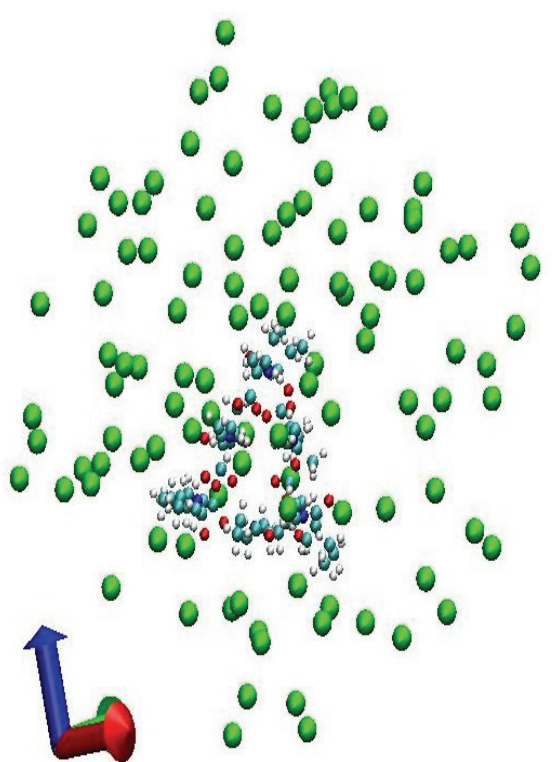

(a)

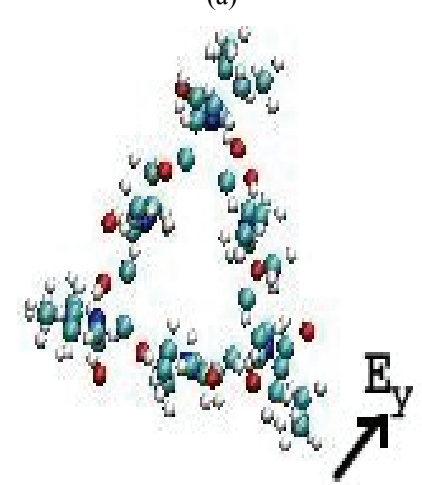

(b)

Figure 3. The valinomycin orientation (a) and the electric field direction (b) for potassium ions. The water molecules are not shown.

changes was fixed in space, so that the valinomycin molecule would be able only to vibrate (oscillate); the directional mobility of the valinomycin molecule was fixed in the initial position. In such conditions, valinomycin's interaction with $\mathrm{K}^{+}\left(\mathrm{Na}^{+}\right)$ions and water molecules happen efficiently in the cavity (ring) region. Figures 4 (a) and (b) shows the equilibrium configuration of the valinomycin $+\mathrm{Na}^{+}$ions surrounded by water molecules; six consequent snapshots show the valinomycin structure with a $\mathrm{Na}^{+}$ion passing through the cavity.

It should be noted that $\mathrm{K}^{+}\left(\mathrm{Na}^{+}\right)$ion passing through valinomycin's ring is not possible for all (arbitrarily) values of the electric field. Namely, for each ion type $\left(\mathrm{K}^{+}\right.$or $\left.\mathrm{Na}^{+}\right)$, a critical electric field value exists under which the ion remains captured (localized) in valinomycin's ring cavity. The MD simulation results presented in Figures 5-8 illustrtate $\mathrm{K}^{+}$ion localization 

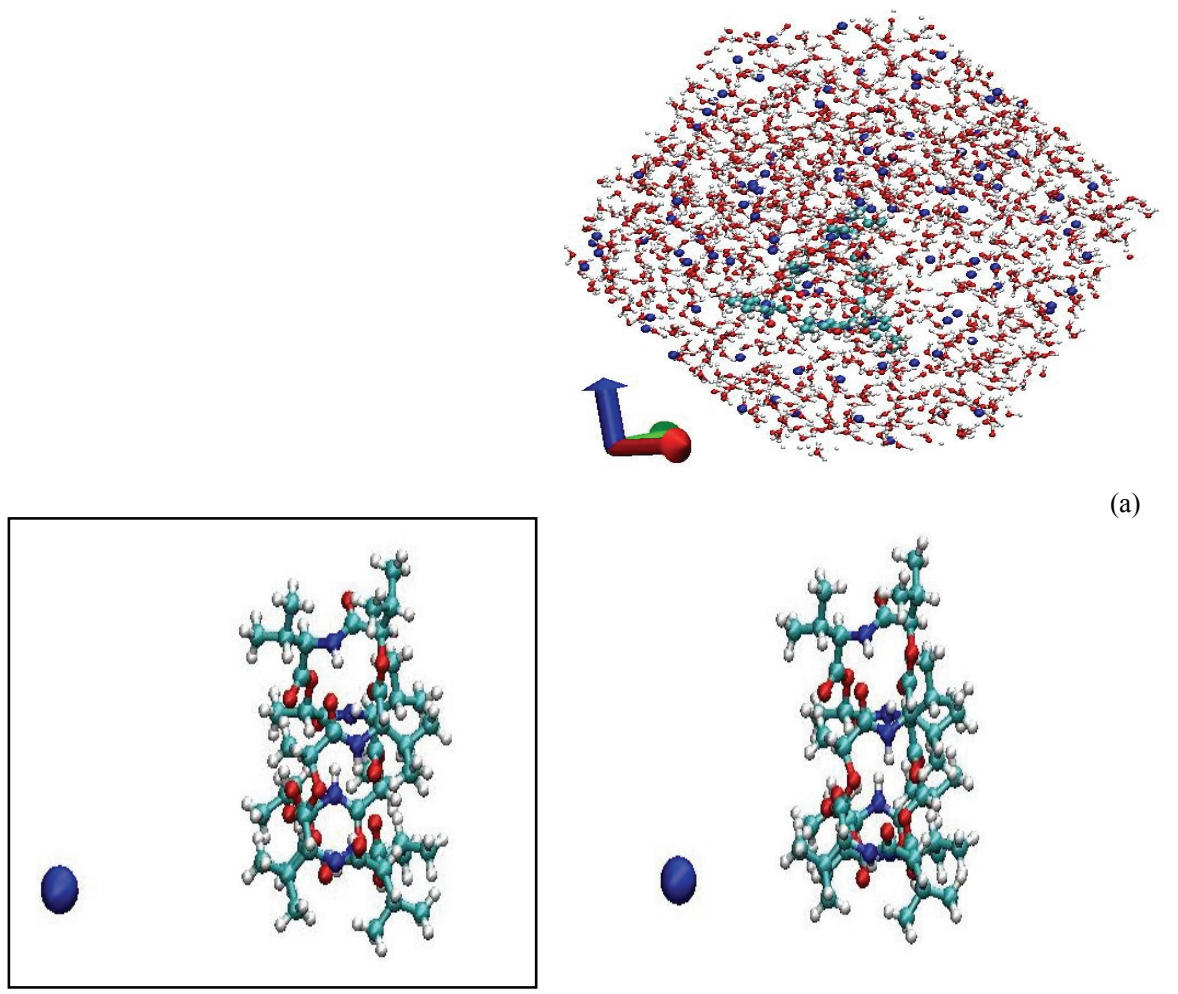

(a)
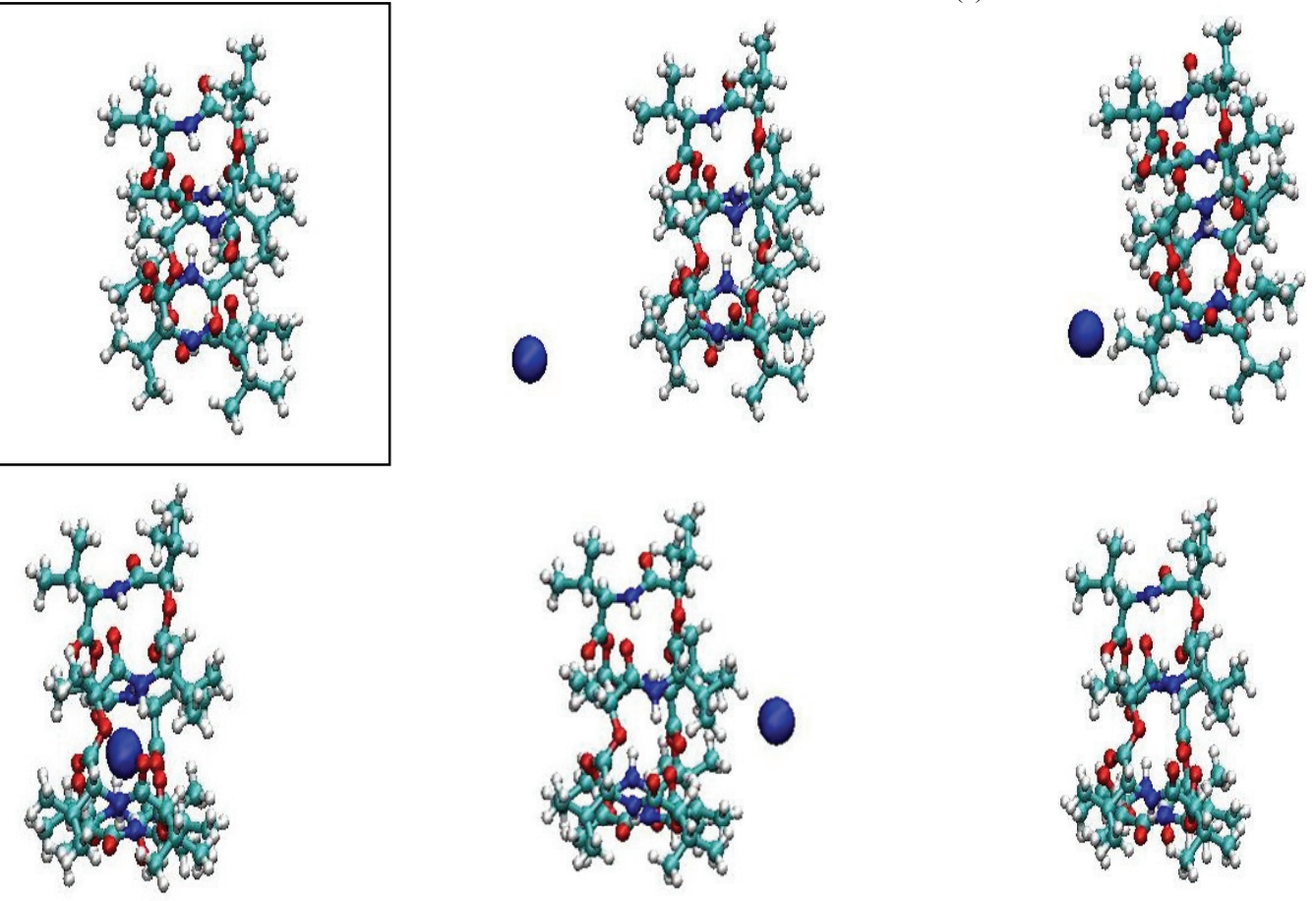

(b)

Figure 4. The valinomycin configuration (a) surrounded by sodium ions (blue spheres) in water. Six consequent configurations of valinomycin and a sodium ion penetrating into the cavity are shown (b). The snapshots correspond to the time moments of $\mathrm{t}=0,1,2$, 3,5 and $10 \mathrm{ps}$ (the electric field is directed from left to right).

(capture) by a valinomycin molecule inside the ring cavity. In Figure 5, the consequent dynamical configurations for valinomycin $+\mathrm{K}^{+}$ion are shown. In Figures 6 (a)-(c), trajectory diagrams are presented for three ions moving outside of valinomycin's localization region. The diagrams in Figures 6(a)-(c) represent motion of ions in a periodic geometry. Figure 7(a) displays the trajectory diagram of a $\mathrm{K}^{+}$ion captured by valinomycin's localization ring. Figure 7(b) shows the consequent configurations of the system in the localization region.

Let us estimate the $\mathrm{K}^{+}\left(\mathrm{Na}^{+}\right)$binding with a valinomycin molecule based on the critical values of the electric field. The simulation results show different critical values for $\mathrm{K}^{+}$and $\mathrm{Na}^{+}$ions. The critical values correlate with the difference in the ion masses $(\mathrm{K} / \mathrm{Na}=39.1 / 23.0)$. A summary of MD simulation results is presented in Table 3 . It is seen that the critical electrical field, under which the ion remains localized inside valinomycin's ring, is about $150 \mathrm{mV}$ for $\mathrm{K}^{+}$and about $90 \mathrm{mV}$ for $\mathrm{Na}^{+}$. The critical values of the electrical field shown in Table 3 can be associated with the chemical binding strength between the ions and the valinomycin molecule. In our estimation of $U_{c r}$ in Table 3, we used the following simple relation: $U_{c r}=E_{c r} d$, where $d \sim 3 \AA$ was chosen as a length of valinomycin's active region (the ring's cross section length).

$$
\mathrm{U}_{\mathrm{cr}}\left(\mathrm{K}^{+}\right) \sim 5 \times 10^{8} \mathrm{~N} / \mathrm{Q} \times 3 \times 10^{-10} \mathrm{~m} \sim 150 \mathrm{mV} ;
$$



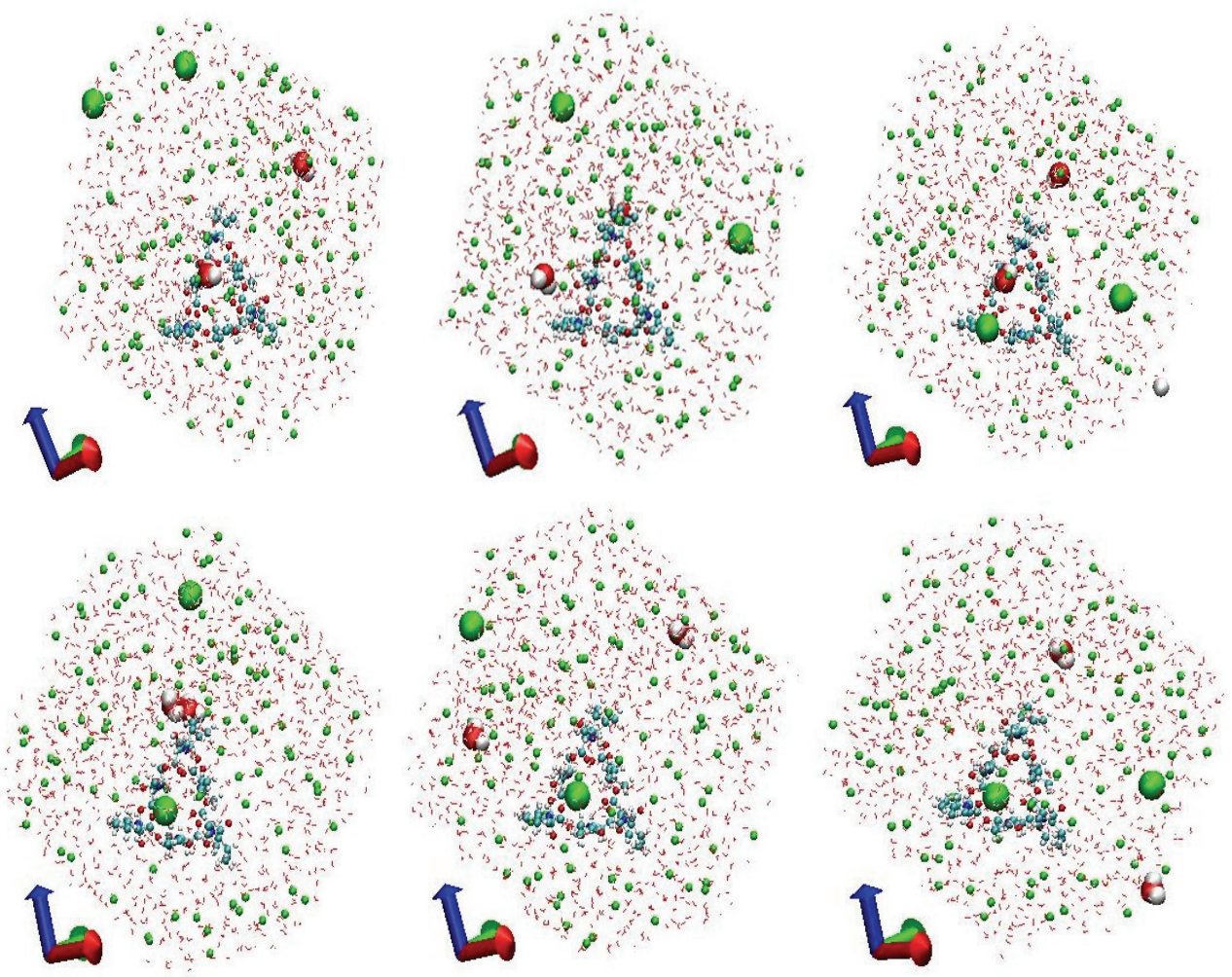

Figure 5. Six consequent configurations of valinomycin with potassium ions (green spheres). The snapshots correspond to the time moments of $\mathrm{t}=0,1,2,3,5$ and $10 \mathrm{ps}$ (views from left to right and from top to bottom).

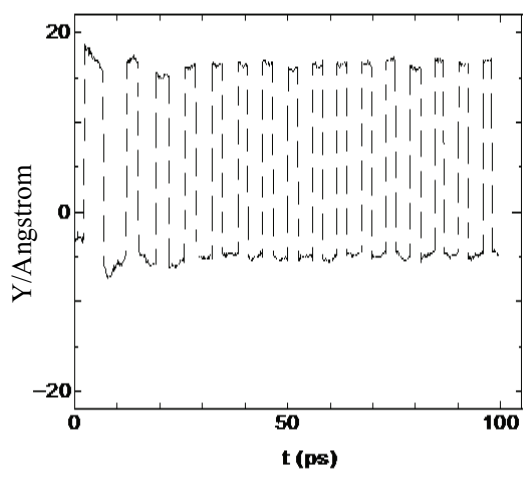

(a)

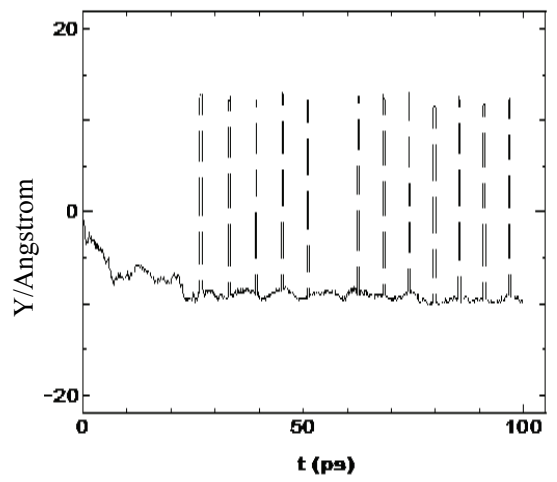

(b)

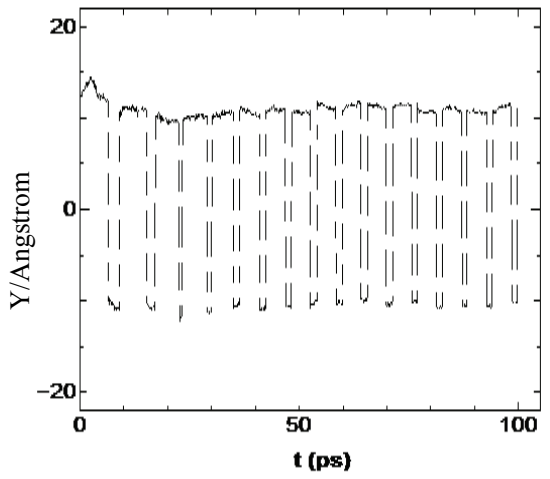

(c)

Figure 6. A trajectory diagram of three potassium ions that are outside of valinomycin's ring (outside of the ion localization in the valinomycin cavity).

$$
\mathrm{U}_{\mathrm{cr}}\left(\mathrm{Na}^{+}\right) \sim 3 \times 10^{6} \mathrm{~N} / \mathrm{Q} \times 3 \times 10^{-10} \mathrm{~m} \sim 90 \mathrm{mV} .
$$

In summary, the external electric field has been used to estimate the strength of two major $\left(\mathrm{K}^{+}\right.$and $\left.\mathrm{Na}^{+}\right)$ion bindings with the valinomycin molecule in water solvent. A stronger valinomycin binding with the potassium ion is clearly observed. It is well known that the valinomycin molecular structure is folded in such a way that its chain conformation efficiently surrounds a metal cation [1-6,8-10,12]. Valinomycin is selective to the $\mathrm{K}^{+}$ion, because it folds in such a way that it forms an almost octahedral structure via its strong (non-polarizable) donors: carbonyl's hydrogen atoms. This structure has to exactly correspond to the $\mathrm{K}^{+}$ion in size.

The ratio of the critical electrical potentials $\mathrm{U}_{\mathrm{cr}}\left(\mathrm{K}^{+}\right)$/ 


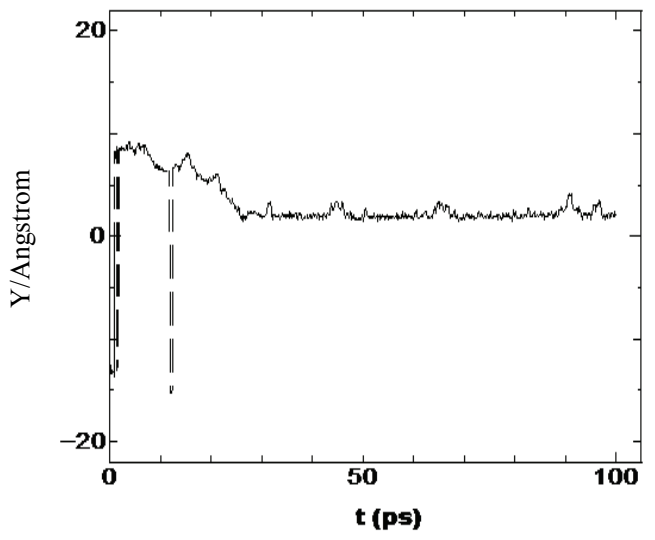

(a)
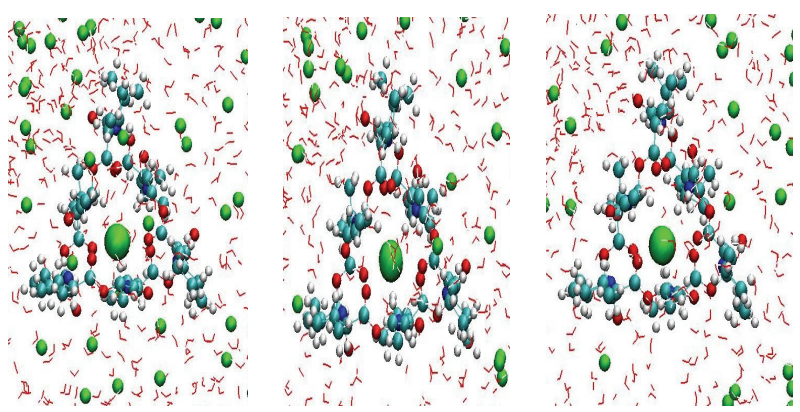

(b)

Figure 7. A trajectory diagram of a potassium ion captured by a valinomycin molecule (a). Three consequent configurations (b) show the ion position inside the valinomycin localization cavity.

Table 3. The values of the critical electric fields for $\mathrm{K}^{+}$and $\mathrm{Na}^{+}$ions.

\begin{tabular}{ccc}
\hline Critical electric field & $\mathrm{K}^{+}$ & $\mathrm{Na}^{+}$ \\
\hline $\mathrm{E}_{\mathrm{cr}}, \times 10^{8} \mathrm{~N} / \mathrm{Q}$ & 5 & 3 \\
$\mathrm{U}_{\mathrm{cr},} \times 10^{-3} \mathrm{~V}$ & 150 & 90 \\
\hline
\end{tabular}

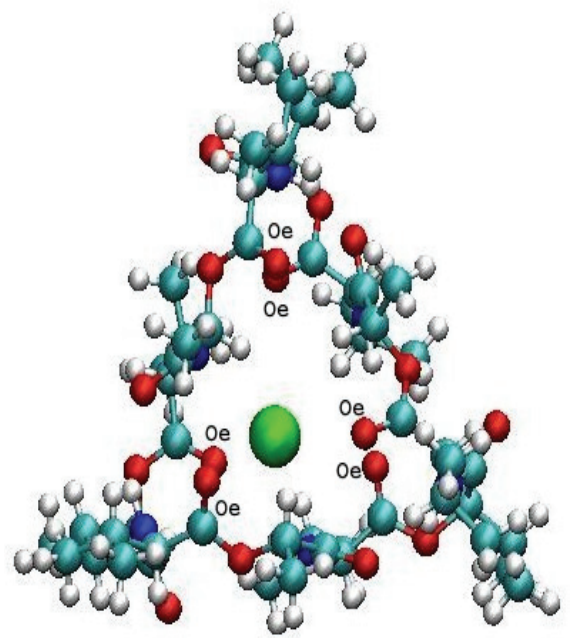

(a)

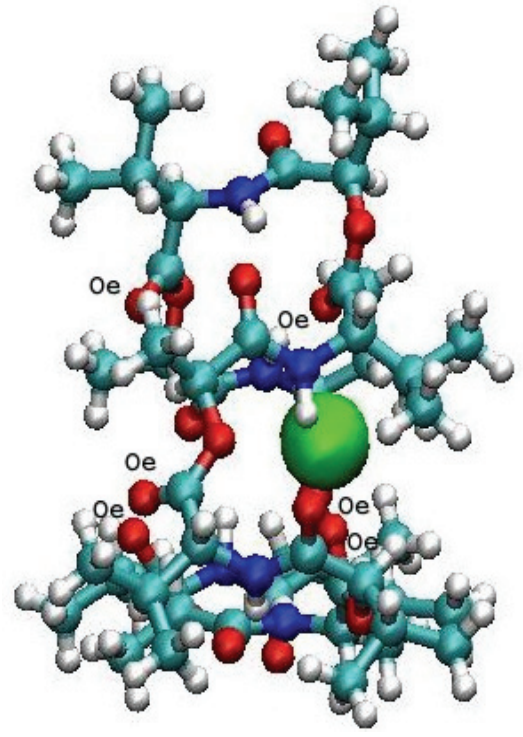

(b)

Figure 8. The valinomycin configuration with potassium ions localized in the ring cavity.

$\mathrm{U}_{\mathrm{cr}}\left(\mathrm{Na}^{+}\right) \sim 1.7$ implies a stronger binding of valinomycin $+\mathrm{K}^{+}$compared to that of valinomycin $+\mathrm{Na}^{+}$, which results in binding energy estimation that $\mathrm{W}\left(\mathrm{K}^{+}\right)>\mathrm{W}\left(\mathrm{Na}^{+}\right)$. The binding for valinomycin $+\mathrm{K}^{+}$is energetically stronger, which correlates well with a number of experimental observations. For example, in experimental salt extraction equilibrium measurements [13], the $\mathrm{Na}^{+}$ $\rightarrow \mathrm{K}^{+}$ion replacement revealed that valinomycin prefers binding $\mathrm{K}^{+}$to $\mathrm{Na}^{+}$by $-5.4 \mathrm{kcal} / \mathrm{mol}$. Other experimental studies of the permeability ratio in lipid membranes [14] show that valinomycin selects $\mathrm{K}^{+}$rather than $\mathrm{Na}^{+}$with a selectivity of about $-6 \mathrm{kcal} / \mathrm{mol}$. The correlation of the simulation results and experimental X-ray crystal structure measurements and related studies of a strongly selective $\mathrm{K}$ channel is straightforward [14-17].

The MD simulation results could be a prerequisite for studying a more complex scenario-for example, protein -ion interactions involving valinomycin together with potassium and sodium ions. It should also be noted that some correlation can be found between the obtained values of the critical electric field strength and the electric potential which is formed in the cell membrane or inside the cell $(\sim 100 \mathrm{mV})$ relative to its surrounding medium. This aspect, however, is worth a more detailed study due to its complexity (in particular, the specifics of the cell membrane like its molecular formation, cross section size, etc. are more complex in terms of structure).

\section{ACKNOWLEDGEMENTS}

This work has been fulfilled under joint collaboration projects of JINR, Russia-Daresbury Laboratory, UK-Keio University, Japan. We 
would like to thank Prof. William Smith for the software support. This work was supported in part by Grant in Aid for the Global Center of Excellence Program for "Center for Education and Research of Symbiotic, Safe and Secure System Design" from the Ministry of Education, Culture, Sport, and Technology in Japan.

\section{REFERENCES}

[1] Steed, I.W. and Atwood, J.L. (2000) Supramolecular chemistry. Wiley.

[2] Doyle, D.A., Cabral, J.M., Pfuetzner, R.A., Kuo, A., Gulbis, J.M., Cohen, S.L., Chait, B.T. and MacKinnon, R. (1998) The structure of the potassium channel: Molecular basis of $\mathrm{K}^{+}$conduction and selectivity. Science, $\mathbf{2 8 0}$ (5360), 69-77.

[3] Forester, T., Smith, W. and Clarke, H.R. (1997) Antibiotic activity of valinomycin. Journal of the Chemical Society Faraday Transactions, 93(4), 613-661.

[4] Ye, Q., Vincze, A., Horvai, G. and Leermakers, F.A.M. (1998) Partial blocking of ion transport at the interface of an ion-selective liquid membrane electrode by neutral surfactants. Experiment and computer simulations. Electrochimica Acta, 44(1), 125-132.

[5] Marrone, T.J. and Kenneth, M.M., Jr. (1995) Molecular Recognition of $\mathrm{K}^{+}$and $\mathrm{Na}^{+}$by valinomycin in methanol. Journal of American Chemical Society, 117(2), 779-791.

[6] Alberts, B., Bray, D., Lewis, J., Raff, M., Roberts, K. and Watson, Y.D. (1994) Molecular biology of the cell. Garland Publishing, A member of the Taylor \& Francis Group, New York.

[7] Smith, W. and Forester, T. (1996) DL_POLY_2.0: A general-purpose parallel molecular dynamics simulation package. Journal of Molecular Graphics, 14(3), 136-141.

[8] Varma, S., Sabo, D. and Rempe, S.B. (2008) $\mathrm{K}^{+} / \mathrm{Na}^{+}$ selectivity in K-channels and valinomycin: Over-coordination vs cavity-size constraints. Journal of Molecular Biology, 376(1), 13-22.
[9] Forester, T.R., Smith, W. and Clarke, J.H.R. (1995) Capture of potassium ions by valinomycin: A molecular dynamics simulation study. Journal of Physical Chemistry, 99(39), 14418-14423.

[10] Eisenman, G., Alvarez, O. and Aqvist, J. (1992) Free energy perturbation simulations of cation binding to valinomycin. Journal of Inclusion Phenomena and Macrocyclic Chemistry, 12(1-4), 23-53.

[11] Smith, W., Forester, T.R. and Todorov, I.T. (2008) The DL poly 2 user manual. STFC Daresbury Laboratory Daresbury, Warrington WA4 4AD Cheshire, Version 2.19 .

[12] van Veggel, C.J.M.F., van Duynhoven, J.P.M., Harkema, S., Wolbers, M.P.O. and Reinhoudt, D.N. (1996) Solidstate structure, dynamical properties in solution and computational studies of a new sodium hemispherand comples. Journal of the Chemical Society Perkin Transactions, 2, 449-454.

[13] Eisenman, G., Aqvist, J. and Alvarez, O. (1991) Free energies underlying ion binding and transport in protein channels: Free energy perturbation simulations of ion binding and selectivity for valinomycin. Journal of the Chemical Society Faraday Transactions, 87, 2099-2109.

[14] Eisenman, G. and Alvarez, O. (1992) Ionic selectivity of proteins: Lessons from molecular dynamics simulations on valinomycin. In: Gaber, B.P. and Easwaran, K.R.K., Eds., Biomembrane Structure and Function - The State of the Art, Adenine Press, Schenectady, 321-351.

[15] Neilson, G.W., Mason, P.E., Ramos, S. and Sullivan, D. (2001) Neutron and X-ray scattering studies of hydration in aqueous solutions. Philosophical Transactions of the Royal Society A, 359, 1575-1591.

[16] Shrivastava, I.H., Tieleman, D.P., Biggin, P.C. and Sansom, M.S.P. (2002) $\mathrm{K}(+)$ versus $\mathrm{Na}(+)$ ions in a $\mathrm{K}$ channel selectivity filter: A simulation study. Biophysical Journal, 83(2), 633-645.

[17] Soper, A.K. and Weckstrom, K. (2006) Ion solvation and water structure in potassium halide aqueous solutions. Biophysical Chemistry, 124(3), 180-191; 39, 1023-1029. 